\title{
Training Assist System of a Lower Limb Prosthetic Visualizing Floor-Reaction Forces Using a Color-Depth Sensing Camera
}

\author{
Kunihiro OGATA $^{\dagger * a)}$, Member, Tomoki MITA ${ }^{\dagger}$, Takeshi SHIMIZU ${ }^{\dagger \dagger}$, and Nobuya YAMASAKI ${ }^{\dagger}$, Nonmembers
}

\begin{abstract}
SUMMARY Some unilateral lower-limb amputees, have through continued exertion, increase the foot reaction force of the sound leg. The asymmetric gait with a prosthetic leg may thus negatively affect the musculoskeletal health of the leg on the healthy side. Therefore, it is important for these amputees to learn how to adjust the balance of each foot load in training. The aim of this study is to develop a training support system visualizing floor-reaction forces using a color-depth sensor. The pose of the entire body of the amputee is measured by the depth sensor, and the floor reaction force is estimated based on Zero Moment Point (ZMP), which is calculated using the center of mass of the amputee. Evaluation experiments of the proposed method were performed and they confirmed the effectiveness of the estimation method and the training with the visualization of reaction force.
\end{abstract}

key words: rehabilitation, visualization, prosthetic leg, zero moment point

\section{Introduction}

If the weight load of the prosthetic limb is not heavy enough for some unilateral lower-limb amputees during gait, the load of the weight that the sound leg withstands increases gradually over time. Chronic pains may be elicited, including fatigue or lumbago, and joint diseases, including pes planovalgus or knee osteoarthritis.

Statistical analysis shows that the amputees consistently loaded the intact limb more than the prosthetic limb, regardless of its role during gait initiation, whether at stance or during swing [1]. Moreover, more joints and muscles of the affected limb are lost owing to higher-level amputations, the greater energy cost and a slower speed [2]. Therefore, it is important for the unilateral amputees to be provided with rehabilitation to balance the left and right weight loads. When the amputees with their prosthetics receive training on how to put load on the prosthetic limb, in most cases, physical therapists and prosthetists verbally instruct the patients on thow to apply the weight load and on the method of loading the prosthetic limb verbally. Correspondingly, the patients with prosthetics adhere to their instructions based

Manuscript received March 12, 2015.

Manuscript revised June 2, 2015.

Manuscript publicized July 28, 2015.

${ }^{\dagger}$ The authors are with Research Institute, National Rehabilitation Center for Persons with Disabilities, Tokorozawa-shi, 3590042 Japan.

${ }^{\dagger \dagger}$ The author is with Rehabilitation Service Bureau, National Rehabilitation Center for Persons with Disabilities, Tokorozawashi, 359-0042 Japan.

* Presently, with the Graduate School of Science and Engineering, Saitama University.

a) E-mail: kuniogata@mail.saitama-u.ac.jp

DOI: 10.1587/transinf.2015EDP7089 on their self-image in a mirror located in the training room, whereby the therapists use two weight scales to instruct the learners to weigh each foot on each scale and adjust the weight load accordingly. The period of learning to walk with their prosthesis is short, and few amputees have the opportunity to be provided with training after social rehabilitation. Therefore, during the early stage of training, the learner's proper adjustment of the weight load of each foot has had an effect on "Activities of Daily Living (ADL)" and the "Quality of Life (QOL)".

The aim of this study is to improve the asymmetrical gait of the amputees and their QOL. Therefore, this work developed a training assist system based on visualizing the gait and weight load of each foot.

\section{Training Assist System Visualizing Floor Reaction Force}

Even though physical therapists and prosthetists instruct the learners using subjective terms, it is important for rehabilitators to show the learners the objective parameters, such as the physiological parameters and physical parameters. Additionally, training-assist systems visualizing objectively several of these parameters have been developed recently, and it is known that their effectiveness is confirmed in regard to the rehabilitation.

\subsection{Past Research of Real-Time Visual Feedback}

Dingwell et al. [3] developed the system for assessing and providing feedback of gait symmetry information in real time to participants walking on a motorized treadmill. The center of pressure shows a representation of this output display. Moreover, real-time visual feedback not only results in an immediate improvement in symmetry, but there are also concomitant reductions in heart rate and oxygen consumption while patients walk at a steady pace on a treadmill [4]. Davis then suggested that visual feedback rehabilitation may serve as a useful tool for amputee patients. Nakagawa et al. [5] developed a prosthetic gait training system that consisted of two load cells that were included in a parallel bar and in prostheses, and visualized the floor reaction forces.

The gait training system known as computer-assisted rehabilitation environment (CAREN) was effective in improving gait performance in a person with a unilateral transfemoral amputation [6]. The use of virtual reality (VR) en- 
vironment based on real-time feedback holds great promise for improving the rehabilitation of individuals with an amputation.

On the other hand, real-time visual feedback was shown to be effective in motor training for cerebral palsy. Sabe et al. developed the new visual feedback device, which measured the load that the patients exert on the handrails, and directly showed this load by using a display [7].

\subsection{Visualizing Load Weight without Force Sensors}

Based on literature [4], the visualization of the floor reaction force is important for the rehabilitation in gait with prosthesis. Therefore, the purpose of this study is the development of a rehabilitation-assist system that visualizes the floor reaction force in real time. However, three-dimensional optoelectronic motion-capture systems and force sensors are prohibitively expensive for clinics for introducing them in daily practice. Additionally, the learners with prosthetics need to use the devices with force sensors or load cells but the measurement area is limited. Gait training programs are prescribed to assist in the rehabilitation process of many clinical conditions. In these cases, low-cost and convenient systems without the need to use force sensors.

Recently, an inexpensive and convenient device that can measure the entire human pose was developed, and the device was used for the rehabilitation.

Clark et al. assessed the concurrent validity of kinematic data recorded using Microsoft's KINECT [8], during a gait retraining session [9]. Llorens et al. [10] presented a study involving chronic stroke patients for the evaluation of the clinical effectiveness of a virtual stepping exercise using skeleton tracking through the KINECT depth sensor. Kitsunezaki et al. [11] developed several applications that used KINECT for physical rehabilitation tests, such as walking motion and joint range, and confirmed the effectiveness of these applications.

The color-depth sensor (KINECT) can extract the entire body pose of a human, so a method to estimate the floor reaction force using the depth data is proposed in this study.

\section{Estimating Floor Reaction Force Based on Entire Pose Information}

When human stands still or walks without a hand reaction force from an environment, an external force from only feet is exerted on the human only from the feet. Therefore, this floor-reaction force equals the inertia force of entire body. This floor reaction force can then be estimated using the time-series data of the pose information of the entire body.

However, the human body is a complex and nonlinear multi-link structure. Therefore, to estimate the floor reaction force in real-time, a calculation method based on a simple dynamic model is proposed in this study.

The outline of the proposal method is shown in Fig. 1. This human body is treated as the simple dynamic model, and the floor-reaction force is estimated based on the model. (a)

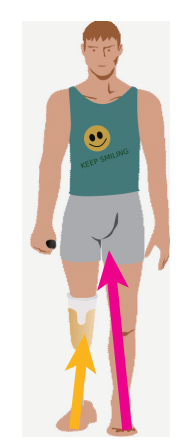

(b)

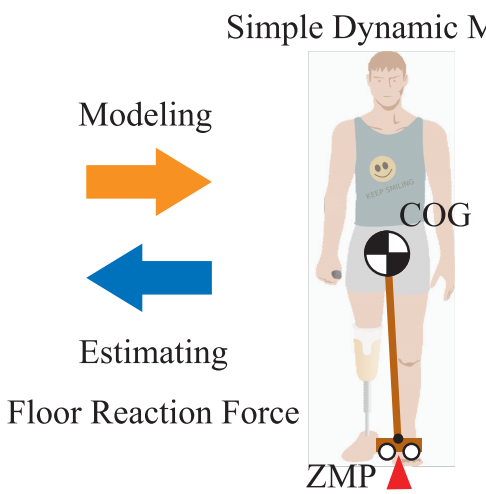

Fig. 1 Mechanism of the proposed system where the floor reaction forces are estimated based on a simple dynamic model.

The detailed algorithm is explained in 3.1 and 3.2.

\subsection{Simple Dynamic Model}

Vukobratovic [12] specified a zero moment point (ZMP) with respect to which the dynamic reaction force at the contact of the foot with the ground does not produce any moment in the horizontal direction, and constitutes the point where the total of horizontal inertia and gravity forces equals zero [13].

This ZMP utilized a pattern generator and a stabilization controller of the bipedal locomotion robot [14]-[16]. It is known that the motion of the humanoid robot based on the walking pattern generator with the ZMP dynamic model is similar to the motion of the human [17]. Therefore, the ZMP dynamic model may represent a part of the human walking motion. In the cases, where the human body is approximated as a simple mass model. The ZMP is represented by Eq. (1).

$$
\left\{\begin{array}{l}
p_{x}=\frac{M g x+M p_{z} \ddot{x}-M(z \ddot{x}-x \ddot{z})}{M g+M \ddot{z}} \\
p_{y}=\frac{M g y+M p_{z} \ddot{y}-M(y \ddot{z}-z \ddot{y})}{M g+M \ddot{z}}
\end{array}\right.
$$

where $(x, y, z)$ denotes the Center of Gravity (COG) position, $M$ is the mass, $g$ is the acceleration due to gravity, $\left(p_{x}, p_{y}\right)$ is the ZMP position. If $p_{z}=0$, Eq. (1) leads to Eq. (2), known as the ZMP equation.

$$
\left\{\begin{array}{l}
p_{x}=x-\frac{z}{\ddot{z}+g} \ddot{x} \\
p_{y}=y-\frac{z}{\ddot{z}+g} \ddot{y}
\end{array}\right.
$$

From Eq. (2), the human body consisted of multi-link structure is represented as simple dynamic model that has only the COG and the ZMP. The COG of the entire body is calculated by the COG position of each body segment, which is calculated based on the literature [18]. 


\subsection{Calculating Floor-Reaction Force Components}

The aim of this paper is to visualize each foot-reaction force. Therefore, only the dynamic model on the lateral plane is treated, and the dynamic model of the $\mathrm{x}$-axis is omitted in this section.

The ZMP can be calculated by the floor-reaction force and the position of each foot represented by Eq. (3).

$$
p_{y}=\frac{f_{R} y_{R f}+f_{L} y_{L f}}{f_{R}+f_{L}}
$$

where $f_{R}$ is the floor-reaction force of the right foot, $f_{L}$ is the reaction force of the left foot, $y_{R f}$ is the position of the right foot, and $y_{L f}$ is the position of the left foot.

The values of $y_{R f}$ and $y_{L f}$ can be obtained from the color-depth sensor and $p_{y}$ can be calculated based on Eq. (2). However, $f_{R}$ and $f_{L}$ are unknown parameters. Therefore, another constraint equation is needed to be set.

The sum of each of the reaction forces equals the inertia of COG. The constrained condition is then set in accordance to the following equation Eq. (4).

$$
f_{R}+f_{L}=F
$$

where $F=M(g+\ddot{z})$. Corresponding, $f_{R}$ and $f_{L}$ are specified based on Eq. (5), which in turn is based on Eq. (3) and Eq. (4).

$$
\left\{\begin{array}{c}
f_{R}=\frac{p_{y}-y_{L f}}{y_{R f}-y_{L f}} F \\
f_{L}=\frac{-p_{y}+y_{R f}}{y_{R f}-y_{L f}} F
\end{array}\right.
$$

The position of each joint can be obtained by the colordepth sensor. However, the acceleration of each body segment is needed to calculate the ZMP.

Both $y$ and $z$ are discretized to allow calculation of $\ddot{y}$ and $\ddot{z}$ based on Eq. (6).

$$
\left\{\begin{array}{l}
\ddot{y}=\frac{y(i)-2 y(i-1)+y(i-2)}{T} \\
\ddot{z}=\frac{z(i)-2 z(i-1)+z(i-2)}{T}
\end{array}\right.
$$

where $T$ is the sampling time and $i$ is the time $(i=2,3,4, \ldots)$.

\subsection{Integrated Value of Floor-Reaction Force}

When the learners stand still, they can adjust the floorreaction force of each foot using the visual feedback, however, during the stepping and walking motion, the reaction forces rise and fall frequently. It is then difficult for the learners to control the force in real time. To understand the floor-reaction forces in real time, it may be effective to show the parameters changing incrementally on the display.

Therefore, integrated values of each foot-reaction force

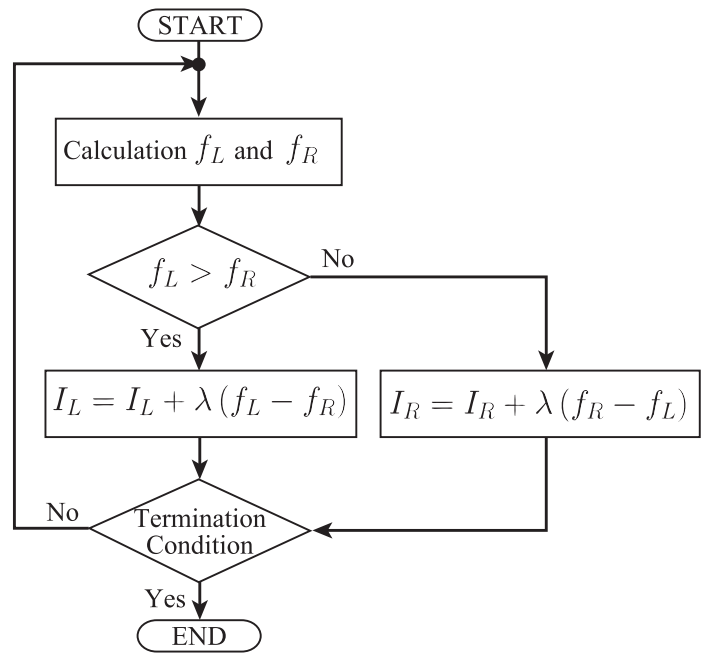

Fig. 2 Flow chart indicating the calculation of the integrated values of each foot reaction force.

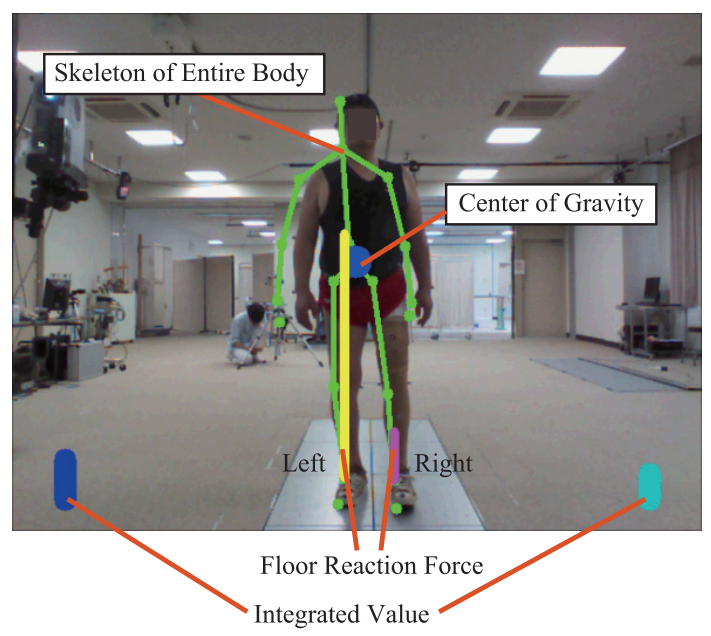

Fig. 3 Display example of the proposed visualization scheme using a color-depth sensor.

are calculated and displayed in real time. The integrated value of the left foot-reaction force is $I_{L}$, and the integrated value of the left foot-reaction force is $I_{R}$. If $f_{L}$ is larger than $f_{R}, I_{L}$ is incremented by $f_{L}-f_{R}$. The flow chart of the algorithm used to calculate the integrated values is shown in Fig. 2.

The display example of visual feedback is shown in Fig. 3. The green lines are the pose of the entire body, the blue circle is the position of the COG, the yellow line is the left reaction force, the magenta line is the right reaction force, the blue line is the integrated value of the left foot, and the cyan line is the integrated value of the right foot. The color data of the camera sensor is displayed as the mirrorreversed image.

\section{Verification Experiments}

To verify the effectiveness of the proposed method, verifi- 


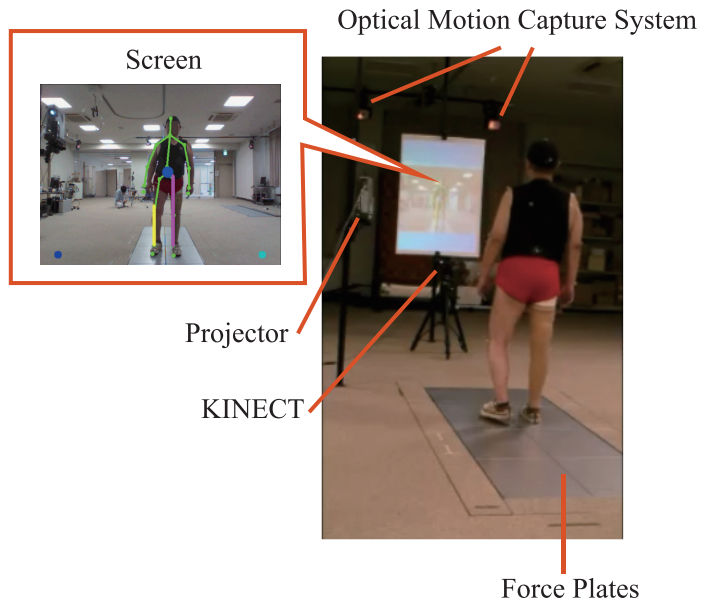

Fig. 4 Overview of the verification experiments.

cation experiments tested on healthy persons and the unilateral leg amputees were performed. In these experiments, the COG and ZMP calculated using the color-depth sensor and the floor-reaction forces were compared against data calculated by an optical motion capture system and force plates. Moreover, the effectiveness of the visual feedback using the proposed system was also verified.

\subsection{Experimental Conditions}

The overview of the experiments is shown in Fig. 4. The KINECT (Microsoft) and the color-depth sensing camera are used to estimate the floor-reaction forces, and the color image data and the floor-reaction forces are shown by the projector. The joints of the entire body are calculated based on the KINECT software developer kit (SDK) [8]. To evaluate the calculation result, the motion data of each participant is measured using the optical motion capture system (motion analysis) and force plates(KISTLER Co. Ltd.).

The participants were six healthy persons and the three unilateral leg amputees, including two trans-tibial amputees and one trans-femoral amputee. The study procedures were approved by the National Rehabilitation Center for Persons with Disabilities Institutional Review Board, and informed consent was obtained from all the participants. In this paper, only parts of the acquired data is processed and presented.

The measurement tasks are "static standing", "stepping motion", and "walking motion" without and with visual feedback. "Static standing" is when the participant keeps standing still for about half a minute. "Stepping motion" is when the participant marches in place for about half a minute. "Walking motion" is when the participant walks about three meters at his/her usual pace for five times.

In the experiments without visual feedback, the participants are instructed to perform each task in a normal way, and in the experiments with visual feedback, the participants are instructed to equalize the left and right floor-reaction forces.

The visualization example where the trans-femoral am-
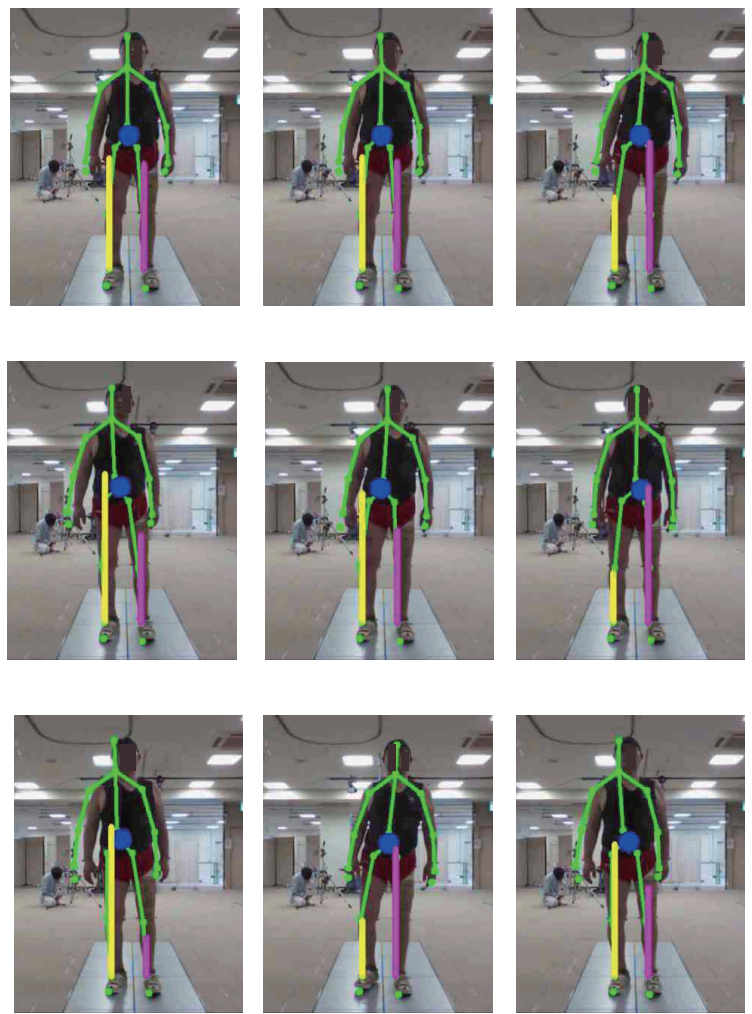

Fig. 5 Snapshots of the trans-femoral amputee as he stepped at locations.

putee stepped at places is shown in Fig. 5. The floor-reaction forces are varied in accordance to the "stepping motion" of the participant, and the right or left force decreased with increases in the other force.

\subsection{Verification of Force Estimation Method}

To evaluate the proposed method, the estimated floorreaction forces of the two healthy participants are shown in Fig. 6 (participant A) and Fig. 7 (participant B). (a) is the calculation result from the force plates, and (b) is the calculation result from the depth-sensing camera.

Comparing the calculation results from the force plates and the depth-sensing camera, the timing of the increase of each floor-reaction force, and the cycle of the trajectories based on the force plates are coincident with the cycle of the calculation results elicited by the proposed method. However, based on Fig. 6(b), the calculation result from the depth-sensing camera yields a steady error. In addition, these trajectories did not decrease to zero.

To analyze the results for which the floor-reaction forces yield the steady error based on the proposed method, the calculated COG trajectories and the ZMP trajectories of the participant A were used as shown in Fig. 8 and Fig.9, respectively..

From Fig. 8, each COG trajectory calculated by the depth-sensing camera is coincident with the trajectories calculated by the motion capture system, and therefore the numerical error of the COG position is sufficiently-small. On 


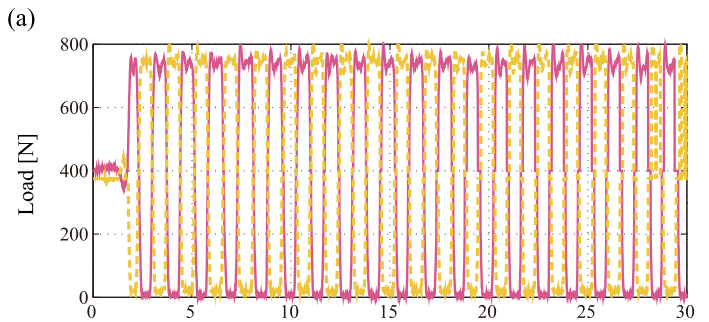

(b)

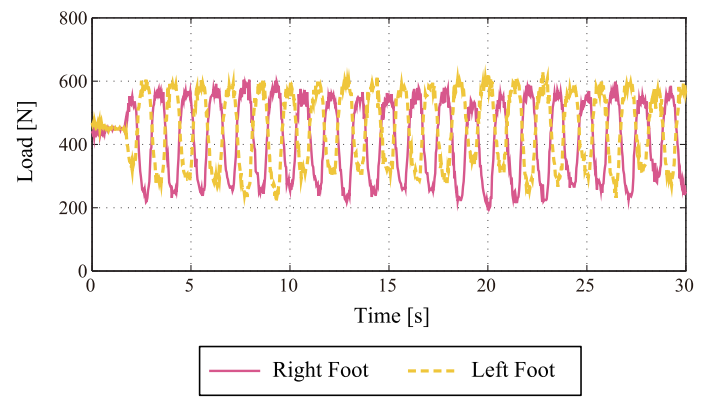

Fig. 6 Comparisons of the (a) calculation results from the force plates, and (b) calculation results from the depth-sensing camera. These are the results of the participant A.

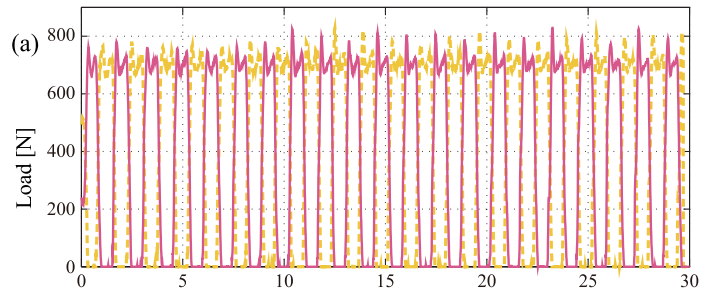

(b)

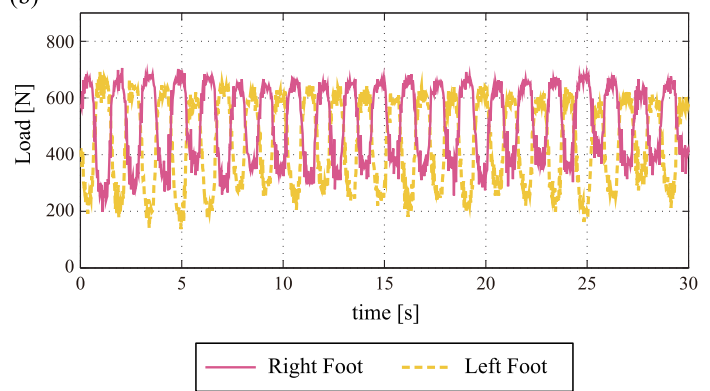

Fig. 7 Comparisons of the (a) calculation results from the force plates, and (b) calculation results from the depth-sensing camera. These are the results of the participant B.

the other hand, from Fig. 9, the error between the ZMP calculated by the depth-sensing camera and the ZMP calculated by the force plates is large when the real ZMP moves to the contact area of each foot. The ZMP is calculated based on the COG position and the COG accelerations are approximated by Eq. (6), yielding the calculation errors of ZMP. Therefore, the floor-reaction forces calculated by the proposed method include the steady error.

These estimation results are associated with the steady error, but the increases and decreases of the right and left floor-reaction forces can be sensed fully. In these cases,
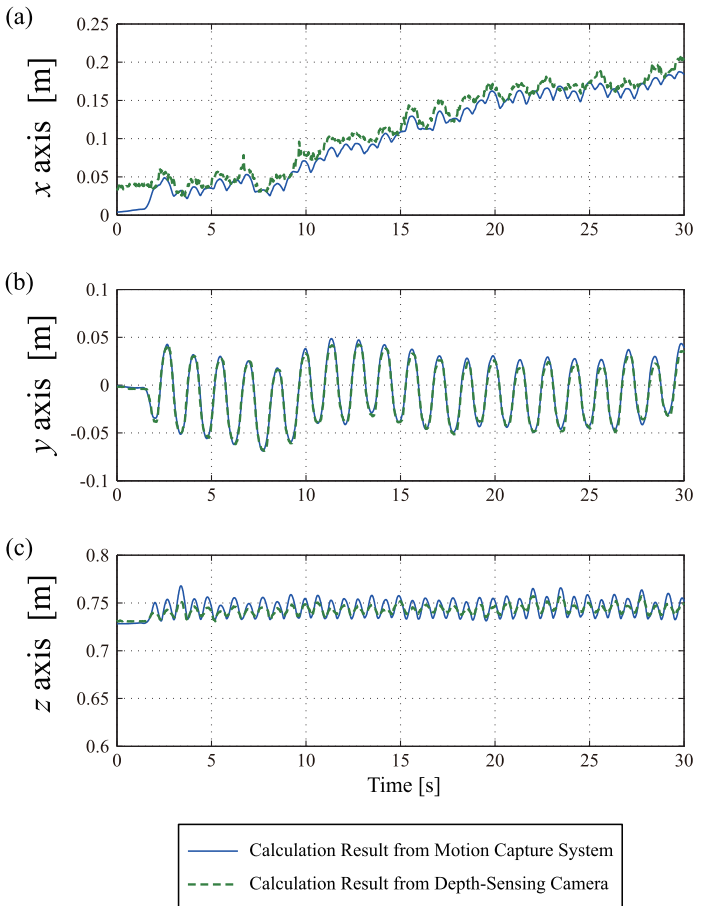

Fig. 8 Calculation results of the center of gravity along the $x, y$, and $z$ axes using the motion capture system and the color-depth sensing camera.

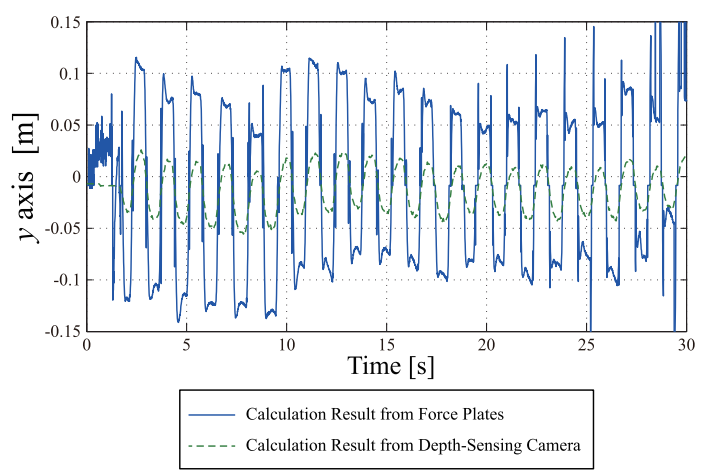

Fig. 9 Estimation results of the zero-moment point on the $y$-axis using force plates and a depth-sensing camera.

these errors are not important for the feedback visualization of the training.

\subsection{Verification of Visualization of Feedback Training}

To verify the effectiveness of the visualization of the feedback training using the proposed method, the amputees adjusted both floor-reaction forces with visual feedback. The experimental results in the case of the trans-femoral amputee under "static standing" are shown in Fig. 10. Figure 10 (a) and (c) depict the actual reaction forces, and Fig. 10 (b) and (d) are the estimated reaction forces. Figure 10-(a) and (b) are the results without visualization, and Fig. 10-(c) and (d) are the results with visualization.

From Fig. 10 (a) and (b), the left reaction force is larger than the right force, and this particular participant wore the 
(a) $[\mathrm{N}]$

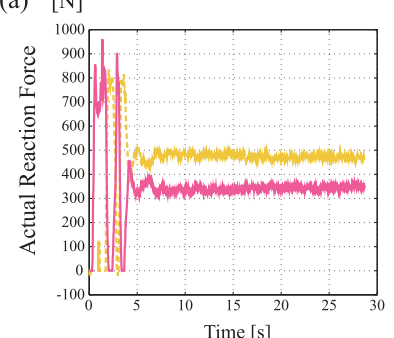

(c)

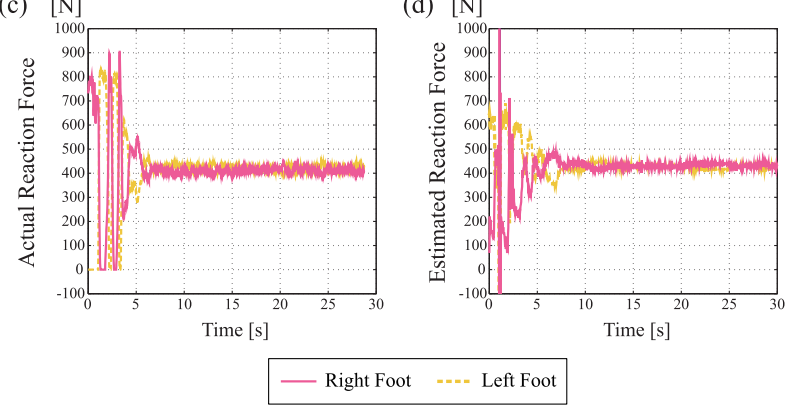

Fig. 10 Evaluation of the effectiveness of the real-time visual feedback using the proposed sensing system for the amputee patient.

right trans-femoral prosthetic limb. This implies that the load of the prosthetic limb was not applied properly. Moreover, the trajectories of the estimated reaction force are similar to the trajectories of the actual reaction force. From Fig. 10 (c) and (d), both left and right reaction forces converged at about the $400[\mathrm{~N}]$, and the participant could thus adjust the left and right reaction forces with the proposed visual feedback training system.

These results have shown that the proposed training system can assist the amputees to train to adjust the floorreaction forces.

\subsection{Integrated Value Results}

To assess on whether the integrated value of the floorreaction force is useful for the lower limb prosthetic training, the integrated floor-reaction forces of the "stepping motion" of the trans-femoral amputee were calculated, and these experimental results are shown in Fig. 11. Figure 11 (a) depicts the estimated reaction forces, and Fig. 11 (b) the integrated values of the floor-reaction forces.

From Fig. 11 (a), the reaction-force of the left foot, that is the unaffected leg, is slightly larger than the right leg that is the prosthetic leg. The walking cycle is about 1.0 [s]. Therefore, it may be difficult for the learner to adjust the reaction force with visual feedback. On the other hand, from Fig. 11 (b), the gap between the left and right reaction forces widened after about fifteen seconds, and the learner could understand the difference between the load of the sound leg and the prosthetic leg.

In this paper, the effectiveness of the visual feedback training using the integrated reaction force was not verified. This verification experiment will be tested in the future.
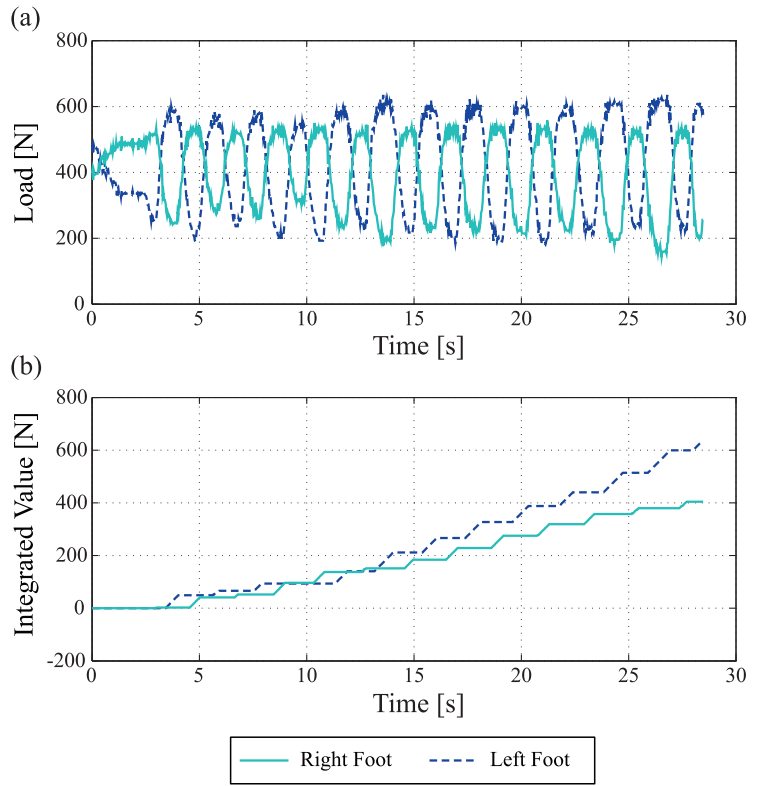

Fig. 11 Computation results of (a) floor-reaction forces, and (b) integrated forces using the proposed algorithm by the participant with a transfemoral prosthesis.

\section{Conclusion}

Some amputees walk with an asymmetrical gait, which may lead to future musculoskeletal degenerative pathologies. Therefore, the rehabilitation that instructs someone how to properly accommodate each foot-reaction force is needed. In this study, the training-assist system visualizing floor-reaction forces was developed and used to verify the effectiveness of the proposed system, and the verification experiments using the integrated value is future work. This training system can visualize the floor reaction force of the amputees using a depth-sensing camera. Given that this system did not use force sensors, it has a low cost and it is convenient. Based on verification experiments, even though the floor-reaction forces had steady errors, the amputees can adjust each floor reaction force using real-time visual feedback, thereby confirming the effectiveness of the proposed method.

In this paper, the training system that visualized floorreaction forces was developed. On the other hand, several musculoskeletal parameters may be more useful for improving the asymmetrical gait. System visualization of several human musculoskeletal data, such as muscle tension information [19], will be collected in future work.

\section{References}

[1] S.A. Rossi, W. Doyle, and H.B. Skinner, "Gait initiation of persons with below-knee amputations: The characterization and comparison of force profiles," Journal of Rehabilitation Research and Development, vol.32, no.2, pp.120-127, 1995.

[2] J.H. Bowker and J.W. Michael, Atlas of limb prosthetics: surgical, prosthetic, and rehabilitation principles, Mosby-Year Book, 1992. 
[3] J.B. Dingwell, B.L. Davis, and D.M. Frazier, "Use of an instrumented treadmill for real-time gait symmetry evaluation and feedback in normal and trans-tibial amputee subjects," Prosthetics and Orthotics Internatiional, vol.20, no.2, pp.101-110, 1996.

[4] B.L. Davis, M. Ortolano, K. Richards, J. Redhed, J. Kuznicki, and V. Sahgal, "Realtime Visual Feedback Diminishes Energy Consumption of Amputee Subjects During Treadmill Locomotion," AAOP, vol.16, no.2, pp.49-54, 2004.

[5] A. Nakagawa, K. Konishi, Y. Nagakura, and S. Morimoto, "Development of a Prosthetic Gait Training System for Geriatric Amputees," ASSISTECH Report 2002, pp.158-163, 2002 (in Japanese).

[6] B.J. Darter and J.M. Wilken, "Gait Training With Virtual Reality-Based Real-Time Feedback: Improving Gait Performance Following Transfemoral Amputation," Physical Therapy, vol.91, no.9, pp.1385-1394, 2011.

[7] R. Sabe, T. Hayashi, and Y. Sankai, "Visual feedback system showing loads on handrails for gait training," 2012 IEEE/SICE International Symposium on System Integration (SII), pp.337-342, 2012.

[8] J. Shotton, A. Fitzgibbon, M. Cook, T. Sharp, M. Finocchio, R. Moore, A. Kipman, and A. Blake, "Real-Time Human Pose Recognition in Parts from Single Depth Images," CVPR '11 Proceedings of the 2011 IEEE Conference on Computer Vision and Pattern Recognition, pp.1297-1304, 2011.

[9] R.A. Clark, Y.-H. Pua, A.L. Bryant, and M.A. Hunt, "Validity of the Microsoft Kinect for providing lateral trunk lean feedback during gait retraining," Gait \& Posture, vol.38, no.4, pp.1064-1066, 2013.

[10] R. Llorens, M. Alcaniz, C. Colomer, and M.D. Navarro, "Balance recovery through virtual stepping exercises using Kinect skeleton tracking: a follow-up study with chronic stroke patients," Studies in Health Technology and Informatics, vol.181, pp.108-112, 2012.

[11] N. Kitsunezaki, E. Adachi, T. Masuda, and J. Mizusawa, "KINECT applications for the physical rehabilitation," 2013 IEEE International Symposium on Medical Measurements and Applications Proceedings (MeMeA), pp.294-299, 2013.

[12] M. Vukobratovic and J. Stepaneko, "On the stability of anthropomorphic systems," Mathematical Biosciences, Mathematical Biosciences, vol.15, pp.1-37, 1972.

[13] S. Kajita, Introduction to Humanoid Robotics, Springer, 2014.

[14] S. Kajita, M. Morisawa, K. Harada, K. Kaneko, F. Kanehiro, K. Fujiwara, and H. Hirukawa, "Biped Walking Pattern Generator allowing Auxiliary ZMP Control," Proceedings of the 2006th IEEE/RSJ, International Conference on Intelligent Robots and Systems, pp.2993-2999, 2006.

[15] T. Sugihara, "Standing Stabilizability and Stepping Maneuver in Planar Bipedalism based on the Best COM-ZMP Regulator," Proceedings of the 2009 IEEE International Conference on Robotics \& Automation, pp.1966-197, 2009.

[16] S. Kajita, M. Morisawa, K. Miura, S. Nakaoka, K. Harada, K. Kaneko, F. Kanehiro, and K. Yokoi, "Biped Walking Stabilization Based on Linear Inverted Pendulum Tracking," Proceedings of the 2010 IEEE/RSJ, International Conference on Intelligent Robots and Systems, pp.4489-4496, 2010.

[17] K. Miura, M. Morisawa, F. Kanehiro, S. Kajita, K. Kaneko, and K. Yokoi, "Human-like Walking with Toe Supporting for Humanoids," Proceedings of the 2011 IEEE/RSJ, International Conference on Intelligent Robots and Systems, pp.4428-4435, 2011.

[18] Human Body Properties Database, http://www.dh.aist.go.jp/ bodyDB/index-e.html (Accessed: 4 Feb. 2015).

[19] A. Murai, K. Kurosaki, K. Yamane, and Y. Nkamura, "Musculoskeletal-see-through mirror: Computational modeling and algorithm for whole-body muscle activity visualization in real time," Progress in Biophysics and Molecular Biology, vol.103, no.2-3, pp.310-317, 2010.

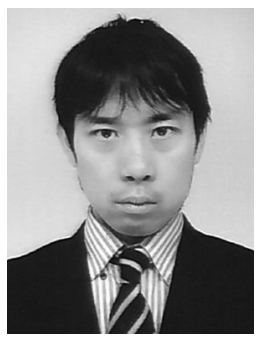

Kunihiro Ogata received the B.E. degrees from College of Science and Engineering Aoyama-Gakuin University, and Master of Information Science and Technology, Ph.D. of Information Science and Technology from The University of Tokyo, in 2006, 2008 and 2012. From 2012 to 2014, he was researcher in Department of Rehabilitation Engineering Research Institute National Rehabilitation Center for Persons with Disabilities. He is currently researcher in the Graduate School of Science and Engineering, Saitama University since 2014. His interests include motion skills, a prosthetic gait and dummy robots. Dr. Ogata is a member of RSJ (Japan), JSME (Japan) and JSAI (Japan).

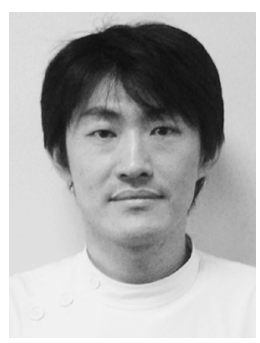

Tomoki Mita received the B.A. degree from Wako University and M. Rehabilitation degree from Tsukuba University, Japan, in 1991 and 2005. He is currently a Prosthetist and Orthotist in the Department of Prosthetics and Orthotics Research Institute National Rehabilitation Center for Persons with Disabilities, since 1997. His interests include fitting of prosthetics sockets for patients with a transfemoral amputation.

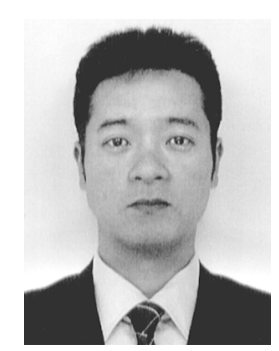

Takeshi Shimizu received the M.Sc. (Health Science) degree in Tsukuba University, in 2012. He is currently a physical therapist in a Division of Daily Living Skills Training for Persons with Physical Disabilities, Department of Daily Living Skills Training, Rehabilitation Services Bureau, National Rehabilitation Center for Persons with Disabilities.

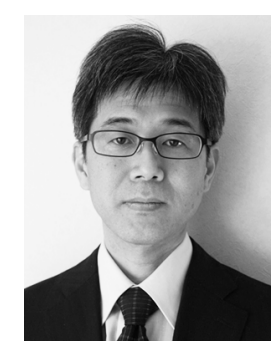

Nobuya Yamasaki received the B.E. degree in Faculty of Engineering, Science University of Tokyo, Japan, in 1997. He is currently a Prosthetist and Orthotist in the Department of Prosthetics and Orthotics Research Institute National Rehabilitation Center for Persons with Disabilities. His interests include Gait analysis of prosthetic leg. Mr. Yamasaki is a member of JSPO (Japan), JAPO (Japan). 